\title{
Normas internacionales de formación para contadores. Bases conceptuales y doctrinarias*
}

\author{
International Training Standards for Accountants. Conceptual and doctrinal bases \\ Padrões internacionais de treinamento para contadores. Bases conceituais e doutrinárias
}

\author{
Miguel Ángel Alegre Britez ${ }^{\text {a }}$ \\ Universidad Nacional de Asunción, Paraguay \\ malegre@eco.una.py \\ ORCID: https://orcid.org/0000-0003-4265-9391 \\ Chap Kau Kwan Chung \\ Universidad del Pacifico, Paraguay \\ ORCID: https://orcid.org/0000-0002-5478-3659
}

DOI: https://doi.org/10.11144/Javeriana.cc22.nifc

Recibido: $07 / 06 / 2021$
Aceptado: $25 / 07 / 2021$
Publicado: $31 / 12 / 2021$

\section{Resumen:}

La formación de los profesionales contables es una necesidad básica para los países que quieren desarrollar su estructura económica y social. El objetivo del trabajo es evaluar las bases conceptuales y doctrinarias de las Normas Internacionales de Formación para Contadores (NIFC). Para la recolección de la información se utilizó una guía de entrevistas compuesta por diez preguntas abiertas cualitativas aplicada a seis expertos en contabilidad y docencia en el área contable de la ciudad de Asunción, Paraguay. El procesamiento de datos se realizó con Atlas. Ti. Los resultados fueron: 1) el aspecto conceptual de las NIFC comprende definición, objetivo, problemática, metodología y resultados de la formación profesional; 2) la base teórica comienza con el concepto contable y los principios del sistema contable, que al final sirven como guía para la formación profesional. Se concluye que en la formación profesional se deben desarrollar los conceptos compromiso, conciencia moral, competencia y comunicación. Los objetivos conceptuales de las NIFC son formar un contador integrado a las normas internacionales, mediante un proceso de aprendizaje basado en el pensamiento científico.

Código JEL: M49.

Palabras clave: Formación profesional, contadores, normas internacionales de formación para contadores.

\section{Abstract:}

The training of accounting professionals is a basic need for countries that want to develop their economic and social structure. The objective of the work is to evaluate the conceptual and doctrinal bases of the International Training Standards for Accountants (ITSA). An interview guide composed of ten qualitative open questions was used for the collection of information, which was applied to six accounting and teaching experts in the accounting area in the city of Asuncion, Paraguay. Data processing was carried out with Atlas. Ti. The results were: 1) The conceptual aspect of the ITSA includes definition, objective, problems, methodology and results obtained in professional training; 2) the theoretical foundation begins with the accounting concept and the principles of the accounting system, which ultimately serve as a guide for professional training. The conclusions are that professional training should developed the concepts of commitment, moral conscience, competence, and communication. The conceptual objectives of the ITSA are to train an accountant integrated to international standards, through a learning process based on scientific thinking. JEL Code: M49.

Keywords: Professional training, accountants, international training standards for accountants.

\section{Resumo:}

A formação de profissionais de contabilidade é uma necessidade básica para os países que desejam desenvolver sua estrutura econômica e social. O objetivo do trabalho é avaliar as bases conceituais e doutrinárias das Normas Internacionais de Treinamento para Contadores (NITC). Para a coleta de informações foi utilizado um roteiro de entrevista composto por dez questões abertas qualitativas, o qual foi aplicado a seis especialistas em contabilidade e ensino da área contábil da cidade de Assunção, Paraguai. O processamento dos dados foi realizado com o Atlas. Ti. Os resultados foram: 1) A vertente conceptual das NITC inclui definição, objetivo, problemas, metodologia e resultados da formação profissional; 2) a fundamentação teórica parte do conceito contábil e dos princípios do sistema contábil que, em última instância, servem de guia para a formação profissional. Conclui-se que na

Notas de autor

\footnotetext{
${ }^{\text {a }}$ Autor de correspondencia. E-mail: malegre@eco.una.py
} 
formação profissional devem ser desenvolvidos os conceitos de comprometimento, consciência moral, competência e comunicação. Os objetivos conceituais das NITC são formar um contador integrado aos padrões internacionais, por meio de um processo de aprendizagem baseado no pensamento científico.

Código JEL: M49.

Palavras-chave: Formação profissional, contadores, normas internacionais de formação de contadores.

\section{Introducción}

Las Normas Internacionales de Formación (NIF) son emitidas por la Federación Internacional de Contadores (IFAC por sus siglas en inglés), organización mundial para la profesión de contador fundada en 1977. La misión de la IFAC es servir al interés público, fortaleciendo de forma continuada la profesión contable en todo el mundo y contribuyendo al desarrollo de economías internacionales fuertes estableciendo y promoviendo la observancia de normas profesionales de alta calidad, impulsando la convergencia internacional de estas normas y tomando postura en el interés público donde la experiencia de la profesión sea más relevante (IFAC, 2020).

La IFAC está compuesta por 159 miembros y asociados en 124 países en todo el mundo, que representan aproximadamente 2,5 millones de contadores en la práctica independiente, industria y comercio, el sector público y la educación (Carrasco, 2007). Ningún otro organismo de contadores en el mundo -y solo unas pocas organizaciones profesionales- tienen el apoyo internacional de amplia base que caracteriza a la IFAC (Abdiel \& Castrellón, 2021). La IFAC mantiene relaciones permanentes con otras organizaciones profesionales y puede cooperar con las organizaciones profesionales en el desarrollo de actividades responsables de promover la formación en contabilidad (Castillo \& Baute, 2021).

Las NIF contribuyen a que los contadores y otros profesionales relacionados con la formación en materia contable puedan llevar a cabo su actividad con garantías de calidad (Juárez, 2021). Las NIF destacan el estándar profesional del auditor independiente y están dirigidas al currículo de las facultades o escuelas de contaduría (Cano \& González, 2021). Las NIF para contadores profesionales prescriben las normas de "buena práctica" generalmente aceptadas en los programas de formación y desarrollo para contadores profesionales (Federación Internacional de Contadores (IFAC, 2008).

Buena práctica se refiere a la existencia de un conjunto generalmente aceptado de normas que los profesionales de la contabilidad deben cumplir para satisfacer sus responsabilidades profesionales (Slosse, 2021). Durante las últimas décadas, buena práctica ha sido traducido al inglés como good practice (GP) y buenas prácticas (BP) (Villanueva, 2016). Las Declaraciones sobre las Prácticas Internacionales de Formación para Contadores Profesionales ayudan en la implementación de la "buena práctica" en la formación y desarrollo de los contadores profesionales, suministrando información acerca de cómo alcanzar los niveles de "buena práctica" o la "mejor práctica" actual (Cantillo, Mendoza, \& De la Hoz, 2021).

Los Documentos Informativos para Contadores Profesionales promueven el debate acerca de las cuestiones de formación y desarrollo que afectan a la profesión contable y presentan resultados o describen situaciones de interés sobre la formación (Ozuna, 2021). La profesión contable tiene por objeto la elaboración de balances o estados financieros y el asesoramiento fiscal y contable a personas naturales, sociedades y negocios en general con el objeto de asesorarles sobre su nivel de liquidez e información para los planes o procedimientos bajo los cuales han optado sus operaciones (Rojas \& Sánchez, 2021). Muchas veces los mismos contadores desconocen las áreas en que necesitan capacitarse, e incluso hay temas conocidos por el contador, pero que no son utilizados correctamente (Rueda, González, \& Avendaño, 2021).

Esta es una de las razones más importantes para que existan las Normas Internacionales de Formación (Moreno, Neiza, \& Cuervo, 2020). Los cambios que se han producido en la profesión contable durante los últimos años han sido, principalmente, consecuencia de las normas internacionales y las buenas prácticas más recientes (Loayza, 2021). 
Las normas internacionales están basadas en la buena práctica adoptada por países pertenecientes a la Federación Internacional de Contadores (Fernández \& Díaz, 2021). El objetivo de las normas internacionales es que un contador profesional formado en cualquier país conozca y aplique correctamente las técnicas o las prácticas contables en sus respectivas jurisdicciones (Egúsquiza, 2001).

Con lo expuesto, se tiene la siguiente pregunta general de la investigación: ¿Cuáles son las bases conceptuales y doctrinarias de las Normas Internacionales de Formación para Contadores? De manera que el objetivo de la investigación es evaluar las bases conceptuales y doctrinarias de las Normas Internacionales de Formación para Contadores.

\section{Métodos}

El enfoque de la investigación es cualitativo y el método es inductivo-deductivo con un diseño fenomenológico. La técnica de recolección de datos fue la entrevista a seis profesionales contables de Asunción de Paraguay los cuales tienen 20 años o más de experiencia en contabilidad y auditoría, así como en la enseñanza superior universitaria. El instrumento de recolección de datos denominado guía de entrevista tuvo diez preguntas abiertas cualitativas. Los datos recolectados se analizaron con el programa Atlas. Ti. Los resultados fueron presentados en forma de preguntas y respuestas con un análisis global de la información recolectada.

\section{Resultados}

A continuación se presentan los resultados más relevantes del estudio.

\section{1) ¿Qué son las Normas Internacionales de Formación para Contadores?}

Entrevistado 1. Las Normas Internacionales de Formación para Contadores están diseñadas para que, a través de ellas, los contadores y/o las empresas beneficien al público. No se trata de un diploma sino de una serie de ciertos requisitos académicos que se deben superar. Los programas de Certificación Internacional de Contabilidad han sido compatibles con las Normas Internacionales de Contabilidad y están diseñados para que los contadores del mundo sean capaces de aplicar estándares internacionales en el trabajo diario. Cada programa requiere una experiencia laboral como contador por al menos dos años, probada y verificada, antes de la concesión del título.

Entrevistado 2. Las Normas Internacionales de Formación para Contadores establecen el currículum para la formación de los profesionales contadores, y están basadas en la buena práctica. El currículum tiene por objeto que se conozcan y apliquen los contenidos más recientes y/o los más básicos de la contabilidad en cada jurisdicción. Por lo tanto, son normas internacionales que están basadas en la buena práctica.

Entrevistado 3. Las Normas Internacionales de Formación para Contadores desean que las personas participen en el desarrollo de la profesión contable, pero no tienen por objeto que sean aplicables en cualquier jurisdicción y no son bases para fines de ninguna jurisdicción. Las normas internacionales son guías para facilitar el control y la aplicación del marco legal y los requisitos de los contadores, lo cual es muy importante para la formación del contador en cada país. La buena práctica requiere también una preparación para ejercer la profesión. No es posible que un contador aplique correctamente los parámetros de la buena práctica si no tiene la formación adecuada.

Entrevistado 4. Las Normas Internacionales de Formación para Contadores son guías académicas y no académicas que establecen el currículum de la formación del contador. Están dirigidas a las facultades o escuelas y no se difundirán a través de diplomados, diplomaturas o especialidades complementarias. Se dice que las Normas Internacionales de Formación para Contadores están basadas en buena práctica, pero desde luego, la buena práctica es una preceptiva aplicable en cada país. 
Entrevistado 5. Las Normas Internacionales de Formación para Contadores son las guías que físicamente establecen qué país aplica qué norma internacional.

Entrevistado 6. Las Normas Internacionales de Formación para Contadores constituyen un conjunto de programas académicos para la selección, el desarrollo y la formación del contador profesional.

2) ¿Cuál es el papel de las Normas Internacionales frente a los requisitos del Estado?

Entrevistado 1. Las Normas Internacionales de Formación para Contadores no son requisitos. Cada profesión está sometida al código o legislación del Estado donde ejerce su actividad. Aunque pueden ser el camino para la legalización de la profesión, no son requisitos u obligatorios. Se ha dicho que las Normas Internacionales de Formación para Contadores son instrumentos de 'eficiencia' pero no se trata de eficacia. Es algo muy distinto.

Entrevistado 2. Las Normas Internacionales de Formación para Contadores van a ser grabadas en libros y certificados por ciertos países, ya que es un requerimiento legal o una normativa que se ha adoptado recientemente. Pero no tienen ningún valor actual. El currículum es la norma que se registra en la biblioteca de cada país y los jóvenes que están en el curso recibirán la certificación de formación en manos del profesor, pero no es un requisito para ingresar a la profesión.

Entrevistado 3. Para obtener un certificado de contabilidad o auditoría se necesita por lo menos prestar tres años de servicio en el ámbito profesional con capacitación adecuada y verificada. Se ha dicho que las Normas Internacionales de Formación para Contadores están basadas en buena práctica, pero desde luego la buena práctica es una preceptiva aplicable en cada país. La certificación internacional no es un requisito sino una medida de efectividad o funcionamiento.

Entrevistado 4. Las Normas Internacionales de Formación para Contadores son guías para la formación del contador profesional. Por más que se den a conocer en una jurisdicción, no son requisitos. Están disponibles para que los profesionales de la contabilidad y la auditoría las utilicen, según sus propios objetivos.

Entrevistado 5. Las Normas Internacionales de Formación para Contadores no son requisitos y su registro no es el fin sino el medio para el desarrollo de la profesión. Los requisitos cuentan con todos los elementos y requisitos para la formación del contador profesional.

Entrevistado 6. Las Normas Internacionales de Formación para Contadores son, por lo tanto, solamente una medida de transparencia o eficiencia institucional.

\section{3) ¿Cuáles son las ventajas de las Normas Internacionales de Formación para Contadores?}

Entrevistado 1. Las ventajas de las Normas Internacionales de Formación para Contadores es que ninguna jurisdicción puede decir que tiene una primacía en la contabilidad. Una jurisdicción no puede decirle a otra cómo realizar los trabajos contables y conseguir el objetivo al que se propone. Esto se ha demostrado en muchos sitios del mundo.

Entrevistado 2. Son las guías que han sido aprobadas por los organismos oficiales, por ejemplo, la OCDE, el FMI, la OIT y otras.

Entrevistado 3. Las Normas Internacionales de Formación para Contadores sirven como una evolución parcial de las Normas Internacionales de Contabilidad. Por lo tanto van a parecerse, pero se sabe que el proceso de desarrollo es diferente en cada país - por razones culturales y estéticas- y está en marcha. En cada país se trabajan la formación del contador y no sólo los objetivos en lo que se refiere a la adecuación, el contexto y los parámetros.

Entrevistado 4. Las Normas Internacionales de Formación para Contadores son una medida muy útil e importante porque contribuyen a desarrollar las Normas Internacionales de Contabilidad y su Asociación Internacional.

Entrevistado 5. Las Normas Internacionales de Formación para Contadores han sido creadas para lograr una adecuación aumentada, es decir, son necesarias en todo el mundo. Su función es lograr la mayor uniformidad de las Normas Internacionales de Contabilidad, así como las Normas Internacionales de Auditoría y sus procedimientos en general. 
Entrevistado 6. Las Normas Internacionales de Formación para Contadores se proponen aumentar el alcance y la calidad del currículum de cualquier país.

4) ¿Cuáles son los retos en la aplicación de las Normas Internacionales de Formación para Contadores?

Entrevistado 1. El principal reto, después de la adopción de las Normas Internacionales de Formación para Contadores, es la flexibilidad y el tamaño del currículum. Las grandes diferencias culturales y sistemas contables entre países hacen necesario un currículum flexible para adecuarlo a cada país. El primer reto -como hemos dicho- es la flexibilidad y el tamaño del currículum. El segundo es que todos los países puedan aplicar las normas internacionales de formación para contadores conjuntamente.

Entrevistado 2. Los miembros de la comunidad académica internacional se han propuesto que aparezcan actualizadas las normas internacionales de educación del contador, pero actualmente no se han elaborado y aún falta mucho trabajo.

Entrevistado 3. La comunidad académica internacional no ha logrado aprobar normas de educación del contador y está trabajando en este tema.

Entrevistado 4. El desarrollo tecnológico contable es importante en todos los países porque se ha logrado realizar los avances de la tecnología contable y su uso en el ámbito de la contabilidad. Esto reduce el tamaño del currículum y hace más fácil su aplicación.

Entrevistado 5. El desarrollo de la tecnología contable y la adecuación del currículum a ella son los retos que tenemos que afrontar.

Entrevistado 6. Se llegará a un mejor desarrollo si se ofrece un currículum más flexible y con mayores resultados. Sin embargo, procurar evitar que se haga un currículum para cada país y se olvide lo importante de la educación y su adaptación institucional.

5) ¿Qué aportan las Normas Internacionales de Formación para Contadores al desarrollo del sector?

Entrevistado 1. Las Normas Internacionales de Formación para Contadores ayudan en la integración de los países del Sistema Contable Mundial. Se necesitan más contadores y menos códigos o tradiciones reguladoras. Las Normas Internacionales de Formación para Contadores son una ayuda para el desarrollo de la profesión.

Entrevistado 2. Las Normas Internacionales de Formación para Contadores ayudan a los países a realizar el cambio de lo que las mismas denominan 'tradiciones locales' a lo que se conoce como el sistema contable mundial. Sirven también para eliminar las barreras que impiden la integración de los países del sector, pues la educación profesional es un factor clave en este proceso.

Entrevistado 3. Las Normas Internacionales de Formación para Contadores ayudan a la integración del sistema contable mundial.

Entrevistado 4. Las Normas Internacionales de Formación para Contadores han sido desarrolladas por la Asociación Internacional de Contabilidad sirven para aumentar la calidad de la formación de la profesión en todo el mundo.

Entrevistado 5. Las Normas Internacionales de Formación para Contadores son una ayuda para el desarrollo del sector. Son normas que se pueden aplicar -según los requerimientos de cada país o modificar a fin de que puedan ser aplicadas en cada país. Este es el primer reto que tenemos que solucionar.

Entrevistado 6. Las Normas Internacionales de Formación para Contadores son un importante instrumento de integración y el alumno puede aprender a formar contadores en cualquier país.

6) ¿Qué se puede esperar de las Normas Internacionales de Formación para Contadores?

Entrevistado 1. Se puede esperar que las Normas Internacionales de Formación para Contadores estén ya aplicadas en varios países. El mundo se está preparando para que todos los países formen sus contadores de acuerdo con las Normas Internacionales de Formación para Contadores.

Entrevistado 2. Las Normas Internacionales de Formación profesional del contador son importantes en el sistema contable mundial porque la educación contable es un requisito indispensable en el crecimiento económico y la consolidación de la democracia internacional. Estas normas sirven como una ayuda para que sean aplicadas por toda la comunidad académica internacional. 
Entrevistado 3. Las Normas Internacionales de Formación para Contadores son importantes en el desarrollo del sector porque describen las características y objetivos de los contadores. Se han aplicado en muchos países del mundo y sirven para la adecuación de la educación profesional.

Entrevistado 4. Las Normas Internacionales de Formación para Contadores, los acuerdos internacionales, los convenios provinciales y nacionales que se han logrado, los acuerdos firmados entre las federaciones y por lo tanto las reglas internas, están contribuyendo al desarrollo de la profesión como una función fundamental del sector en el desarrollo sustentable.

Entrevistado 5. Se puede esperar que las Normas Internacionales de Formación para Contadores tengan efectos positivos en la profesión. En unos cuantos años estas normas ya habrán sido elaboradas por las Asociaciones Contables y aplicadas en varias universidades.

Entrevistado 6. Con las Normas Internacionales de Formación para Contadores aparecerán los primeros contadores del mundo que tendrán la misma formación. Las Normas Internacionales de Formación para Contadores deben ser aplicadas necesariamente a todos los países.

7) ¿Cuál es el papel y los retos de los gremios contables en el desarrollo del sector?

Entrevistado 1. Los gremios contables deben extender sus funciones para que el sistema contable mundial pueda ser más internacional. Deben hacer más campañas de formación internacional con el fin de lograr la adecuación a las Normas Internacionales de Formación para Contadores y a los acuerdos internacionales para la certificación profesional del sector.

Entrevistado 2. Los gremios contables están contribuyendo al desarrollo de su sector porque han confirmado, con sus propias acciones, lo que se venía diciendo desde hace mucho tiempo: un profesional bien formado es un contador con una sola línea de tiempo y con un mínimo de competencias.

Entrevistado 3. Los gremios contables deben promover la formación profesional y tener una solución a los problemas del sector. Debe ofrecer papeles para formar a los contadores.

Entrevistado 4. El papel de los gremios contables es importante porque ellos han desarrollado, y ahora están aplicando por todo el mundo las Normas Internacionales de Formación para Contadores.

Entrevistado 5. El papel de los gremios contables en el desarrollo del sector es importante porque han apoyado las propuestas de formación profesional que contribuyen a la integración del sistema contable mundial.

Entrevistado 6. El papel y las funciones de todos los gremios son importantes para el desarrollo del sector. Como contadores debemos tener a mano toda la información que nos pueda servir para desarrollar nuestro trabajo. Debemos, además, fortalecer las comunidades académicas para que se formen contadores.

8) ¿Cuál es su opinión de la evolución y los retos de la profesión en las próximas décadas?

Entrevistado 1. El desarrollo de la profesión será inmejorable. La profesión tendrá que seguir preparándose para el futuro. La política y el Estado deben aprovechar su potencial. El desarrollo tecnológico contable y la tecnología financiera son una gran ayuda.

Entrevistado 2. Los modelos contables y las normas deben ser evolucionados para ajustarse al sistema contable mundial. El uso de la tecnología debiera también considerarse como un requisito en los procesos de formación profesional.

Entrevistado 3. La profesión seguirá desarrollándose, aún más con la incorporación de las Normas Internacionales de Formación para Contadores y los acuerdos internacionales para la certificación profesional. Entrevistado 4. Los retos de la profesión en los próximos años serán el trabajo en equipo y la comunicación.

Entrevistado 5. El sector continuará desarrollándose con las Normas Internacionales de Formación para Contadores. Los gremios contables finalmente están aceptando que hay una profesión contable única y que todos los países deben formar contadores con un mismo nivel de competencias.

Entrevistado 6. Los retos de la profesión son los mismos en todos los países. El sector continuará desarrollándose para integrarse con el resto del mundo y ser capaz de ayudar a cada país a mejorar su situación económica. 


\section{9) ¿Qué se puede esperar del futuro del sistema contable internacional?}

Entrevistado 1. El futuro del sistema contable mundial es promisorio. Cambiará poco a poco y seguirá siendo integrado con el resto del mundo.

Entrevistado 2. El sistema contable mundial se debe formar en todos los países con las mismas competencias. El avance tecnológico continuará desarrollando el sector.

Entrevistado 3. El sistema contable mundial se puede esperar que integre a cada contador en el desarrollo de cada país.

Entrevistado 4. El sistema contable mundial se debe enfocar a ser perdurable y con un mínimo de competencias.

Entrevistado 5. El sistema contable internacional continuará desarrollándose con las Normas Internacionales de Formación para Contadores. Una estrategia de desarrollo será la cooperación e integración entre los países.

Entrevistado 6. Se debe esperar que el sistema contable mundial se integre con el resto del mundo y que ayude a los países a desarrollarse económicamente.

10) ¿Cuál es el papel y los retos del profesional contable en el futuro de la profesión?

Entrevistado 1. Los profesionales contables tienen que formarse para ser competentes y para saber hacerse valer ante todos los sectores de la administración pública. Deben tener dotes que se adapten a las necesidades de cada sistema de gobierno, pero en todo caso deben tener una preparación científica profesional.

Entrevistado 2. Los profesionales contables deben seguir formándose para ser competentes, tener dotes para las acciones humanitarias y ser eficientes.

Entrevistado 3. Los profesionales contables deben estar preparados para la variedad de roles actualmente desempeñados por ellos. Deben ser capaces de adaptar sus actividades a las necesidades de cada sistema de gobierno.

Entrevistado 4. Los profesionales contables deben seguir formándose y desarrollándose para cubrir todas las necesidades. Es importante que puedan adaptarse a la variedad de roles actualmente desempeñados por ellos.

Entrevistado 5. Los profesionales contables deben seguir formándose y desarrollándose para conocer los nuevos retos de la sociedad.

Entrevistado 6. Los profesionales contables deben seguir formándose para conocer los nuevos retos de cada país. Deben continuar formando empleados públicos y privados y convertirse en líderes de opinión en beneficio del desarrollo económico del país.

\section{Discusión}

Las respuestas muestran cómo los seis entrevistados perciben las características de las Normas Internacionales de Formación para Contadores (NIFC); la mayoría manifestaron que son guías. Dos entrevistados consideran que son programas académicos y dos entrevistadores opinan que son un conjunto de programas académicos. Esto se explica, en parte, por el hecho de que una vez recibidas pueden ser modificadas o incorporadas a las actividades académicas o profesionales, esto concuerda con Cantillo et al. (2021) y Cano \& González (2021) en cuanto a la necesidad de un mejoramiento en los programas académicos de la formación del contador público.

En cuanto a los requisitos que están relacionados con la ley y las NIFC, los entrevistados consideran dichas Normas como guías y establecimientos de currículum, pero no como requisitos legales. Además, mencionaron que las Normas Internacionales de Contabilidad propician la adecuación de la contabilidad, y estas no van a ser opuestas a las NIFC. Por lo tanto, ambos instrumentos son complementarios y en el ámbito profesional son una medida para la adecuación. Esto difiere de Egúsquiza (2001), quien considera que se debe reglamentar específicamente las normativas contables a nivel del país en que se las utiliza. 
No obstante, casi todos coincidieron en que el principal reto es la flexibilidad en el currículum y la aplicación de éste, debido al número de países con los que hay correspondencia en todos los aspectos. Por tal efecto, la adaptación del currículum a cada país es una medida para garantizar el adecuado desarrollo de las NIFC. Asimismo, se vio que las Normas Internacionales de Formación para Contadores aportan al desarrollo del sector al permitir a los países integrarse en el sistema contable mundial, además de servir como un mecanismo para la integración profesional, lo cual está en línea por lo sugerido por Abdiel \& Castrellón (2021).

Cabe señalar que los entrevistados, en su mayoría, estuvieron de acuerdo en que la aplicación de las NIFC o el desarrollo de un currículum que sirva como base para la formación contable mundial es lo que se puede esperar. Y en cuanto a los gremios contables, parecieron coincidir en que este órgano constituye una parte importante en el desarrollo del sector. Por lo cual, para que las NIFC sean aplicadas es necesario que los gremios apoyen la formación profesional. Según la IFAC (2008) todos los organismos involucrados en la contabilidad deben trabajar armónicamente.

En general, los entrevistados estuvieron de acuerdo en que el sector seguirá desarrollándose gracias al trabajo en equipo y la comunicación, a la integración del sistema contable mundial y a las NIFC. Se espera también que el sistema contable mundial se desarrolle a través de la integración, es decir, que todos los países puedan entenderse y promover una cooperación. Los Gremios Contables están aceptando el hecho de profesionales con mínimos de competencias, ya que es lo que ahora se ve necesario para permitir la adecuación de los países al sistema contable mundial. Por último, los profesionales contables deberían seguir formándose para ser competentes y adaptarse a las necesidades de cada sistema de gobierno individualmente. El papel del profesional contable en el futuro será mucho más importante debido a la importancia que tienen en el desarrollo económico y social, dichas ideas están acordes con Fernández \& Díaz (2021) y Juárez (2021), en lo concerniente a la formación continua del profesional contable.

\section{Conclusiones}

1) La sociedad en su conjunto no está dispuesta a dejar solos sus asuntos económicos y éticos. La sociedad demanda de los profesionales contables una adecuada formación profesional y los roles que deben desempeñar en el futuro.

2) Los entrevistados expresaron la intención de mejorar su formación y su preparación en concordancia con la evolución del sistema contable internacional. Por lo tanto, se debe trabajar en coordinación entre los distintos sectores e instituciones del país, y con el propósito de que las normas internacionales de formación profesional pasen a pensamiento común.

3) Los profesionales contables consideran que son importantes para el desarrollo económico y social del país. En este sentido, deben mejorar su capacitación profesional y tener una forma de pensamiento común para lograr un balance exitoso en su desarrollo profesional y social.

4) Los retos del sistema contable internacional son el desarrollo tecnológico, la integración económica mundial, los nuevos roles del profesional contable en la sociedad y la evolución de los principios éticos.

5) Las implicaciones de la cultura con la moral tienen relación en cuanto al sistema educativo contable en que el contador debe mantener una actitud crítica y tener una moral intachable, lo cual redundará en beneficio de la cultura educativa y un mejoramiento de la formación del contador.

6) La investigación fue cualitativa con diseño fenomenológico, por lo cual se buscó la percepción de los expertos solamente a efectos de mejorar el conocimiento sobre el objeto de estudio.

7) El Estado debe integrarse continuamente en los procesos de innovación educativa de la contabilidad para asegurar que el contador tenga la formación adecuada y actualizada para ofrecer sus servicios eficientemente. 


\section{Consideraciones éticas}

Durante la elaboración del trabajo de investigación se respetaron los derechos de autor mediante las citaciones respectivas, se cuidó el medio ambiente y se tuvieron en cuenta la diversidad de opiniones de los autores citados.

\section{Contribución de los autores}

Chap Kau Kwan C. (coautora) contribuyó en el resumen, introducción y métodos del trabajo. Miguel Ángel Alegre B. (autor principal) contribuyó en resultados, discusión, conclusiones y referencias del trabajo.

\section{Financiación}

El trabajo tuvo financiación propia de los autores.

\section{Conflictos de interés}

En la presente investigación no existió conflicto de intereses con ninguna persona física ni jurídica.

\section{Referencias}

Abdiel, H., \& Castrellón, X. (2021). El contador financiero en la auditoría forense. Revista FAECO sapiens, 4(1), 58-71. https://doi.org/10.48204/j.faeco.v4n1a5

Cano, A., \& González, W. (2021). El discurso ético de lo ecuánime en la formación del contador público colombiano con visión mundial. Revista CIES Escolme, 12(1), 175-191. http://www.escolme.edu.co/revista/index.php/cie s/article/view/328

Cantillo, J., Mendoza, A., \& De la Hoz, A. (2021). Revisión bibliográfica de los planteamientos académicos para mejorar la calidad de la educación contable en Colombia. ECONÓMICAS CUC, 42(2), 01-21. https://doi.or g/10.17981/econcuc.42.2.2021.Econ.5

Carrasco, C. (2007). La tecnología de la información en el currículo de la formación del contador público. Quipukamayoc, 14(27), 89-94. https://doi.org/10.15381/quipu.v14i27.5385

Castillo, Y., \& Baute, L. (2021). Sistema de tareas docentes integradoras: Contribución a la formación ética profesional de los estudiantes contadores. Revista Conrado, 17(80), 216-222. https://conrado.ucf.edu.cu/index.php/conra do/article/view/1834

Egúsquiza, C. (2001). Importancia de la investigación científica en ciencias financieras y contables. Quipukamayoc, 8(15), 61-67. https://revistasinvestigacion.unmsm.edu.pe/index.php/quipu/article/view/5687/4919

Federación Internacional de Contadores - IFAC (2008). Normas Internacionales de Formación 2008. Federación Internacional de Contadores. https://www.ifac.org/system/files/downloads/Spanish_Translation_Normas_In ternacionales_de_Formacion_2008.pdf

Federación Internacional de Contadores - IFAC (2020). La Federación Internacional de Contadores (IFAC). Antecedentes. Federación Internacional de Contadores. https://www.ifac.org/system/files/publications/files/E S_A4_IFAC_Background_Info.pdf

Fernández, N., \& Díaz, N. (2021). La enseñanza de las NIIF en la formación del profesional contable de San Martín. Tesis de grado en contabilidad, Universidad Peruana Unión. http://200.121.226.32:8080/handle/UPEU/4295 
Juárez, V. (2021). La perspectiva ontológica en la construcción curricular de la formación del contador público. Tlamelaua: Revista de Ciencias Sociales, 14(49), 01-08. https://dialnet.unirioja.es/servlet/articulo?codigo=783523 0

Loayza, J. (2021). Experiencias en la producción científica de los estudiantes de la carrera de contabilidad y auditoría de diferentes universidades del Ecuador. Tesis de grado para ingeniero en contabilidad y auditoría, Universidad Técnica de Machala. http://repositorio.utmachala.edu.ec/handle/48000/16661

Moreno, L., Neiza, E., \& Cuervo, J. (2020). Construyendo una estrategia para la formación pedagógico-didáctica del estudiante de Contaduría Pública de la Universidad de Antioquia. Adversia, 25, 1-21. https://revistas.udea.edu .co/index.php/adversia/article/view/344885

Ozuna, L. (2021). La Contabilidad y sus orígenes, caso ciudad de Pilar, Paraguay. Ciencia Latina Revista Cientifica Multidisciplinar, 5(2), 2319-2334. https://doi.org/10.37811/cl_rcm.v5i2.437

Rojas, W., \& Sánchez, A. (2021). Pensando la Contaduría Pública en su ejercicio profesional: Contextos y presupuestos. Innovar, 31(80), 141-152. https://doi.org/10.15446/innovar.v31n80.93671

Rueda, G., González, J., \& Avendaño, W. (2021). El proceso de enseñanza del programa contaduría pública y su relación con las matemáticas. Mundo FESC, 11(21), https://www.fesc.edu.co/Revistas/OJS/index.php/mundofesc/art icle/view/635

Slosse, C. (2021). Una mirada sobre el presente y el futuro de la actividad profesional desarrollada por el auditor externo de estados contables. Audit.AR, 1(1), 35-40. https://doi.org/10.24215/27188647e005

Villanueva, C. (2016). Measuring the TIC impact on the accounting profession teaching. https://alicia.concytec.gob.pe /vufind/Record/1609-8196_d0761c4cf639856bddfcadc13f3c4725/Description

\section{Notas}

* Artículo de investigación

Licencia Creative Commons CC BY 4.0

Para citar estar articulo: Alegre, M. A., \& Kwan, C. K. (2021). Normas internacionales de formación para contadores. Bases conceptuales y doctrinarias. Cuadernos de Contabilidad, 22. https://doi.org/10.11144/J averiana.cc22.nifc 